\title{
SINGLE ANTENNA TIME REVERSAL ADAPTIVE INTERFERENCE CANCELLATION
}

\author{
José M. F. Moura, Yuanwei Jin, Dan Stancil, Jian-Gang Zhu, Ahmet Cepni, Yi Jiang, and Ben Henty \\ Carnegie Mellon University \\ Department of Electrical and Computer Engineering \\ 5000 Forbes Avenue, Pittsburgh, PA 15213 \\ moura@ece.cmu.edu
}

\begin{abstract}
This paper presents the time reversal adaptive interferer canceller (TRAIC), a novel algorithm that uses time reversal techniques to cancel the presence of interferers. TRAIC is developed for broadband signals and a single emitting antenna. Experimental tests in the electromagnetic domain show the viability and the power of TRAIC.
\end{abstract}

\section{INTRODUCTION}

Multipath can deteriorate dramatically the detection performance in radar/sonar problems or in digital communication systems. On the other hand, matched field processing (MFP), [1], and blind channel equalization in wireless communications are just examples where, by the use of appropriate models, multipath can be used to advantage to increase the detection performance or the user capacity of the system. For example, in simple terms, MFP in detection or source localization in underwater acoustics solves an inverse problem (source detection or location) by stepping through a sequence of forward problems, where in each forward problem the unknown location of the source is postulated at each one of potential positions. Practical implementation of MFP implies the solution of the wave equation for each forward problem assuming a given channel velocity propagation profile and given boundary conditions. This is computationally demanding and requires good knowledge of the environmental conditions, both of which make MFP an expensive, sensitive solution for many practical problems. Time reversal (TR), known in optics as phase conjugation, provides a very good alternative to MFP, since it avoids the detailed modeling of the channel, while still providing the potential gain from matching to the propagated field, rather than matching to the original transmitted wavefield. In a sense, time reversal provides the actual channel Greens' function, in contrast with MFP where the channel Greens' function is computed from the model.

This work is funded by the Defence Advanced Research Project Agency through the Army Research Office grant \# W911NF-04-1-0031.
In the last decade, time reversal has been used to increase resolution by exploiting scattering and multipath in inhomogeneous channels. Fink and collaborators demonstrated super-resolution focusing in the ultrasound domain, [2], see their work with controlled ultrasonic experiments in water tanks. More recently large-scale acoustics experiments in the ocean have confirmed the resolution ability in real acoustic propagation environments of time reversal, [3]. There is a growing literature on time reversal in these acoustic and ultrasound fields, as well as on studies of time reversal in random environments and in several applications domains, including medical imaging.

Our work is concerned with exploitation of time reversal in the electromagnetic domain for both focusing and detection. In particular, this paper develops an interferer canceller that can be used in highly cluttered radar detection problems.

\section{INHOMOGENEOUS CHANNEL}

We assume a simplistic discrete time model: a single active antenna (in transmit mode) radiates a wideband signal $s(t) \leftrightarrow S\left(\omega_{q}\right), \omega_{q}=\omega_{0}+q \Delta_{\omega}, q=0 \cdots Q-1$; the channel, with multiple (possibly large number of) scatterers, has impulse response and system function $h(t) \leftrightarrow H(\omega)$. The goal of this paper is to develop an algorithm that adaptively cancels the effect of the interferers (scatterers) using filtered time reversal. The algorithm presented in this paper can be generalized to more complicated and realistic conditions.

After scattering, the signal $r(t)$ received at the antenna (in receive mode), ignoring noise, is the convolution of the channel impulse response $h(t)$ with the transmitted signal. In the frequency domain, it is given as follows,

$$
R\left(\omega_{q}\right)=H\left(\omega_{q}\right) S\left(\omega_{q}\right), \quad q=0, \cdots, Q-1
$$

where $R\left(\omega_{q}\right)$ and $H\left(\omega_{q}\right)$ are the Fourier coefficients of the received signal $r(t)$ and the channel $h(t)$ at the $q$ th frequency bin, respectively. Stacking Eqn. (1) in a vector form 
yields

$$
\begin{aligned}
\mathbf{r} & =\left[R\left(\omega_{0}\right) \cdots R\left(\omega_{Q-1}\right)\right]^{T} \\
& =\left[S\left(\omega_{0}\right) H\left(\omega_{0}\right), \cdots, S\left(\omega_{Q-1}\right) H\left(\omega_{Q-1}\right)\right]^{T} \\
& =\mathbf{S h} \\
\text { where } \mathbf{h} & =\left[H\left(\omega_{0}\right), \cdots, H\left(\omega_{Q-1}\right)\right]^{T} \\
\mathbf{S} & =\operatorname{diag}[\mathbf{s}] \\
\mathbf{s} & =\left[S\left(\omega_{0}\right), \cdots, S\left(\omega_{Q-1}\right)\right]^{T}
\end{aligned}
$$

\section{TIME REVERSAL}

In the usual time reversal experiments, the received signal is stored, delayed by a sufficiently large delay (time window) $T$, time reversed, which in the frequency domain corresponds to phase conjugation, and finally retransmitted. Ignoring the delay $T$, the time reversed signal is given by

$$
\begin{aligned}
\mathbf{r}_{\mathrm{TR}} & =\mathbf{r}^{*} \\
& =\left[S^{*}\left(\omega_{0}\right) H^{*}\left(\omega_{0}\right), \cdots, S^{*}\left(\omega_{Q-1}\right) H^{*}\left(\omega_{Q-1}\right)\right]^{T}(3)
\end{aligned}
$$

$\equiv \alpha$ and $\tau_{l}=l \tau, l=0, \cdots, L-1$. Then, the sums in (9) give rise to (discrete) sinc functions

$$
\left|\alpha^{T} \mathbf{u}\left(\omega_{q}\right)\right|^{2}=|\alpha|^{2}\left|\frac{\sin \left(\omega_{q} \tau / 2\right) L}{\sin \left(\omega_{q} \tau / 2\right)}\right|^{2} .
$$

For $\tau$ small, $L$ large, and $\omega_{q} \tau / 2$ small, these (discrete) sinc functions can be approximated by $L$, so that

$$
\mathbf{z} \approx|\alpha|^{2} L^{2}\left[S^{*}\left(\omega_{0}\right), \cdots, S^{*}\left(\omega_{Q-1}\right)\right]^{T}=|\alpha|^{2} L^{2} \mathbf{s}^{*},
$$

i.e., the time reversed signal backscattered by the channel and received at the antenna is focused, and, except for an attenuation and for time reversal, it replicates the original signal as desired.

\section{TRAIC}

We now develop TRAIC, the time reversal adaptive interference canceller algorithm. TRAIC has exactly the opposite goal of time reversal. While, as mentioned at the end of section 3, in time reversal, we retransmit a delayed and time reversed replica $\mathbf{r}_{\mathrm{TR}}$ of the received signal to refocus the original signal at the antenna, the goal with TRAIC is to minimize the backscattered signal that reaches the antenna after retransmission. We achieve this by rshaping the spectrum of the time reversed signal $\mathbf{r}_{\mathrm{TR}}$. This is useful to cancel the interference caused by undesired scatterers in the channel.

Let $\mathbf{g}=\left[G\left(\omega_{0}\right), \cdots, G\left(\omega_{Q-1}\right)\right]^{T}$ and $G=\operatorname{diag}[\mathbf{g}]$. The signal to be retransmitted is a reshaped version of $\mathbf{r}_{T R}$

$$
\mathbf{y}=G \mathbf{r}_{T R}
$$

The residue clutter signal, i.e., the returned signal after $\mathbf{y}$ is backscattered by the channel is given by, excluding noise,

$$
\begin{aligned}
\mathbf{x}_{c} & =\operatorname{diag}\left[H\left(\omega_{0}\right), \cdots, H\left(\omega_{Q-1}\right)\right] \mathbf{y} \\
& =\left[\begin{array}{c}
G\left(\omega_{0}\right)\left|H^{*}\left(\omega_{0}\right)\right|^{2} S^{*}\left(\omega_{0}\right) \\
\vdots \\
G\left(\omega_{Q-1}\right)\left|H^{*}\left(\omega_{Q-1}\right)\right|^{2} S^{*}\left(\omega_{Q-1}\right)
\end{array}\right]
\end{aligned}
$$

We design the reshaping filter $G$ such that

$$
G_{\mathrm{opt}}=\arg _{G} \min \left\|\mathbf{x}_{c}\right\|^{2}
$$

subject to the constraint

$$
\|\mathbf{y}\|^{2}=E_{s} .
$$

Theorem 1 The optimization problem (12) is given by

$$
\left|G_{\text {opt }}\left(\omega_{q}\right)\right|^{2}=\frac{k_{0}^{2}}{\left|H\left(\omega_{q}\right)\right|^{4}\left|S^{*}\left(\omega_{q}\right)\right|^{2}}
$$




\section{EXPERIMENTS}

$$
k_{0}^{2}=\frac{E_{s}}{\sum_{q=0}^{Q-1}\left|H\left(\omega_{q}\right)\right|^{-2}}
$$

Proof: Notice that

$$
\left\|\mathbf{x}_{c}\right\|^{2}=\sum_{q=0}^{Q-1}\left|H\left(\omega_{q}\right)\right|^{4}\left|S^{*}\left(\omega_{q}\right)\right|^{2}\left|G^{*}\left(\omega_{q}\right)\right|^{2}
$$

Recall the inequality

$$
a_{1}+a_{2}+\cdots+a_{n} \geq n \sqrt[n]{a_{1} a_{2} \cdots a_{n}}
$$

provided that $a_{1}>0, \cdots, a_{n}>0$. Equality holds when $a_{1}=a_{2}=\cdots a_{n}$. Hence,

$$
\left\|\mathbf{x}_{c}\right\|^{2} \geq Q \sqrt[Q]{\prod_{q=0}^{Q-1}\left|H\left(\omega_{q}\right)\right|^{4}\left|S^{*}\left(\omega_{q}\right)\right|^{2}\left|G^{*}\left(\omega_{q}\right)\right|^{2}}
$$

Equality holds when, for $q=0, \cdots, Q-1$,

$$
\left|H\left(\omega_{q}\right)\right|^{4}\left|S^{*}\left(\omega_{q}\right)\right|^{2}\left|G^{*}\left(\omega_{q}\right)\right|^{2}=k_{0}^{2} \in \mathcal{R}^{+}
$$

This leads to

$$
\forall q:\left|G_{\text {opt }}\left(\omega_{q}\right)\right|^{2}=\frac{k_{0}^{2}}{\left|H\left(\omega_{q}\right)\right|^{4}\left|S^{*}\left(\omega_{q}\right)\right|^{2}}
$$

The constant $k_{0}$ is chosen to satisfy the constraint (13), which leads directly to (15). This completes the proof.

Therefore, the residue clutter is given by

$$
\mathbf{x}_{c}=\left[\begin{array}{c}
G\left(\omega_{0}\right)_{\mathrm{opt}}\left|H\left(\omega_{0}\right)\right|^{2} S^{*}\left(\omega_{0}\right) \\
\vdots \\
G\left(\omega_{Q-1}\right)_{\mathrm{opt}}\left|H\left(\omega_{Q-1}\right)\right|^{2} S^{*}\left(\omega_{Q-1}\right)
\end{array}\right]
$$

Without loss of generality, if we choose

$$
G\left(\omega_{q}\right)_{\mathrm{opt}}=\frac{k_{0}}{\left|H\left(\omega_{q}\right)\right|^{2} S^{*}\left(\omega_{q}\right)}
$$

we will have

$$
\mathbf{x}_{c}=k_{0}[1, \cdots, 1]^{T}
$$

The physical meaning of this choice of $G\left(\omega_{q}\right)$ is clear. The reshaping filter is equivalent to a zero-forcing filter that whitens the clutter response, which can then be subtracted from measurements obtained by returned time-reversed echoes. This interference cancellation scheme fully utilizes the operational advantage of time reversal, being a simple phase conjugation in the frequency domain. A more thorough study of TRAIC in detection and localization will be published elsewhere.
To test the focusing and the time reversal adaptive interference canceling (TRAIC) algorithm experimentally, we carried out a sequence of experiments in the electromagnetic domain. We performed a series of transmission measurements through a $9 \mathrm{~m}$ length of $30.5 \mathrm{~cm}$ diameter HVAC duct with caps on both ends. This environment was chosen because its highly reverberant nature enables time reversal focusing to be observed with relatively modest signal bandwidths $(\approx 20 \mathrm{MHz})$. Both the duct and caps are made of galvanized steel. Transmissions were made between $3 \mathrm{~cm}$ monopole probes spaced $3.3 \mathrm{~m}$ in the central region of the duct. Following [4], a $100 \mathrm{~ns}$ pulse centered at $2.45 \mathrm{GHz}$ was generated using an Agilent E4433B digital signal generator with dual baseband arbitrary waveform generators. After transmission through the duct, the signal was downconverted to a $20 \mathrm{MHz}$ center frequency and received with an Agilent $89610 \mathrm{~A}$ vector signal analyzer. Both the $I$ and $Q$ streams of the impulse response were captured. As mentioned in the begining of this section, this system is used to demonstrate both time-reversal focusing and nulling by the TRAIC algorithm.

For focusing, these streams were time-reversed using off-line processing, and the $Q$ stream was additionally multiplied by $(-1)$ to account for phase conjugation of the carrier. The time-reversed baseband waveforms are shown in Figure 1. These time-reversed $I$ and $Q$ streams were then programmed into the digital signal generator and retransmitted through the duct. The receiving probe was located in a longitudinal slot along the duct, allowing its range to be adjusted. Figure 2 shows the temporal and spatial focusing of the received pulse. The target location for focusing is the $15 \mathrm{~cm}$ point along the slot. The height of the focused peak exhibits a clear maximum at the target location for focusing.

To demonstrate nulling by TRAIC, we discuss a time version of the shaping filter. We express it in the time domain. This was done by following the same procedure used for focusing, but additionally finding the point along both the $I$ and $Q$ signals before and after which equal powers were transmitted, and multiplying the leading segment only by $(-1)$. Although crude, this is a very simple way to obtain experimentally the rotated orthogonal version of the signal that is received after transmitting the delayed and time reversed version of the signal that is received after the original transmission, as desired in the TRAIC algorithm. Since, as argued at the end of section 3, convolution with the channel response should collapse in space and time the timereversed signals into a version of the narrow pulse originally transmitted, the physical principle is to alter alternate segments of the signal so that the contributions add destructively instead of constructively. Figure 2 shows the temporal and spatial nulling of the received signal using the 
TRAIC algorithm. In this Figure, the target location at $15 \mathrm{~cm}$ exhibits a clear minimum peak amplitude, demonstrating time-reversal nulling.

\section{CONCLUSION}

Time reversal simplifies the signal processing algorithms in highly reverberant environments where multipath is prevalent by enabling the constructive use of the multipath. Its implementation is, at least theoretically, relatively simple. A signal is transmitted by an antenna. The received signal after scattering by the channel is recorded, delayed, time reversed, which in the frequency domain is simply phase conjugation, and then retransmitted. The signal captured at the antenna after this time reversal signal is scattered by the channel is focused in space and time. This phenomena has been well documented with experimental studies in acoustics and ultrasound. Focusing by time reversal in the electromagnetic domain is less well studied. We believe that our work in [5] is one of the first, if not the first, experimental verifications of focusing in the electromagnetic (EM) domain, see also [4]. Time reversal is essentially a "multipath" based phenomena, i.e., the focusing results from the temporal diversity induced by the multipath. To emphasize this fact, in this paper, we have considered the extreme case of a single antenna. We have illustrated in section 3 that, in fact, if the multipath is rich enough, the channel scattering does focus the time reversed signal back at the receiving antenna.

The paper introduces the time reversal adaptive interference canceler (TRAIC) algorithm. Again, we choose to describe TRAIC in the extreme case of a single antenna to illustrate that, under a sufficiently rich multipath environment and with a broadband signal, we can, by time reversal, cancel out the interference effects of scatterers in the channel. TRAIC can be recast in the time domain and applied with several antennas rather than a single antenna.

In section 5, we demonstrate experimentally in the EM domain both the focusing effect and the nulling of the interferences using TRAIC when we have a single antenna. The multipath environment is recreated with an HVAC duct capped at both extremes. This provides a rich multipath environment. Figures $1-2$ show a nitid focused spot, see Figure 1, where it should focus and a nitid null effect, see Figures 2 . We are currently applying TRAIC to detection by time reversal in highly cluttered radar environments.

\section{REFERENCES}

[1] A. B. Baggeroer, W. A. Kuperman, and P. N. Mikhalevsky, "An overview of matched field methods in ocean acoustics," IEEE J. of Oceanic Engineering, vol. 18, no. 4, pp. 401-424, April 1983.

[2] M. Fink, "Time reversed acoustics," Physics Today, vol. 50, no. 3, pp. 34-40, 1997.
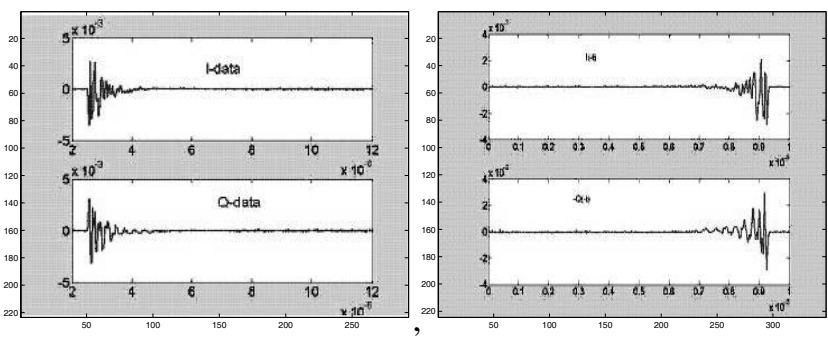

Fig. 1. Left: $I$ and $Q$ in duct; right: time-reversed I and Q.
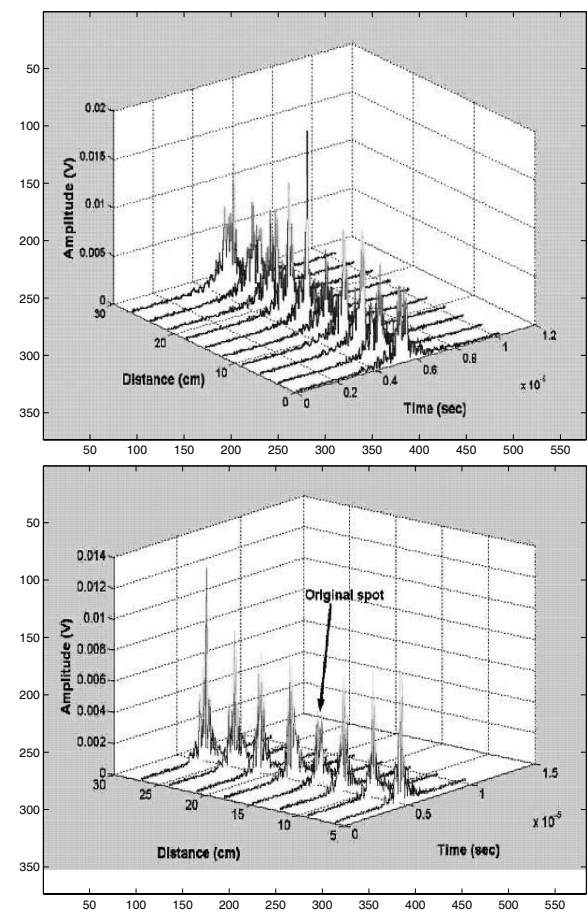

Fig. 2. Target focal location is $15 \mathrm{~cm}$ along scale of slot. Top: Focusing in space and time is apparent; bottom: TRAIC: time-reversal nulling at the target location.

[3] H. C. Song, W. A. Kuperman, W. S. Hodgkiss, T. Akal, and C. Ferla, "Iterative time reversal in the ocean," J. Ac. Soc. of America, vol. 105, no. 6, pp. 3176-3184, June 1999.

[4] G. Lerosey, J. de Rosny, A. Tourin, A. Derode, G. Montaldo, and M. Fink, "Time reversal of electromagnetic waves," Physical Review Letters, vol. 92, pp. 194301, May 2004.

[5] Benjamin E. Henty and Daniel D. Stancil, "Multipath enabled super-resolution for RF/microwave communication using phase-conjugate arrays," Physical Review Letters, 2004. 